\title{
COMPARATIVE STUDY OF SUPERSONIC NOZZLES
}

\author{
MD. Safayet Hossain ${ }^{1}$, Muhammad Ferdous Raiyan ${ }^{2}$, Nahed Hassan Jony ${ }^{3}$ \\ ${ }^{1}$ Department of Mechanical Engineering, CUET, Chittagong, Bangladesh \\ ${ }^{2}$ Department of Mechanical Engineering, CUET, Chittagong, Bangladesh \\ ${ }^{3}$ Department of Mechanical Engineering, CUET, Chittagong, Bangladesh
}

\begin{abstract}
In this experiment, comparative flow analysis of two different nozzles has been performed. The analysis has been performed according to the shape of the nozzles by keeping the same input parameters. The experiment has been carried out in two preliminary steps. First one includes modeling and CFD analysis and the later part is about comparing their different properties. For this analysis, two dimensional axisymmetric nozzle geometries were drawn in Solid Works and CFD analysis is done using Fluent. The basic difference between these two nozzle geometries is their outlet divergence angle, whereas the inlet crosssectional area, throat cross-sectional area and nozzle length are exactly same. These two nozzle geometries were drawn having outlet divergence angle $10^{\circ}$ and $20^{\circ}$ respectively. Velocity, pressure and temperature distribution on both nozzles have been studied to take the final decision. From analysis, it is clearly observed that the nozzle having outlet divergence angle $20^{\circ}$ gives higher exit velocity with Mach number of 5.62 whereas the nozzle with outlet divergence angle $10^{\circ}$ gives an exit velocity with Mach number of 4.31. Besides, lower temperature distribution and lower pressure distribution were observed in the nozzle with outlet divergence angle $20^{\circ}$ throughout the expansion zone and nozzle with outlet divergence angle $10^{\circ}$ exhibits higher temperature and pressure throughout the expansion zone. As the nozzle with divergence angle $20^{\circ}$ gives higher exit velocity, it is the better one between these two nozzles.
\end{abstract}

Keywords: Convergent-divergent nozzle, CFD, ANSYS Fluent, Outlet divergence angle, SolidWorks.

\section{INTRODUCTION}

In the area of fluid mechanics, nozzle is occupying a major portion and it is one of the most important fields of fluid mechanics. Application of nozzle can be found in a wide variety of places. From aircraft propulsion to fuel sprayer, application of nozzle is seen in industrial, automobile, aerospace and in many other sectors. Supersonic nozzle is the nozzle which provides an output velocity with a supersonic speed. The input velocity can be supersonic or subsonic but the output will always be supersonic in case of a supersonic nozzle. The main purpose of this experiment is to compare different parameters of two supersonic nozzles having different divergence angle. The purpose is to investigate the differences in the parameters of these nozzles due to change in outlet divergence angle. Both nozzles which are investigated in this analysis are convergentdivergent nozzles.

When fluid travels through the converging portion of the nozzle towards the throat, it experiences a pressure drop and a rise in velocity. There is also a drop in the enthalpy or total heat of the fluid. The drop of enthalpy is not utilized to some external work rather it is converted into kinetic energy. In the divergent portion (from throat to outlet), there is a further drop in pressure and a further rise in velocity. Again there is a drop in enthalpy or total heat of the fluid which is converted to kinetic energy.

For a steady flow process in nozzle,

$$
\mathrm{h}_{1}+\frac{\mathrm{v}_{1}^{2}}{2}=\mathrm{h}_{2}+\frac{\mathrm{v}_{2}^{2}}{2}+\text { losses }
$$

Neglecting the losses,

$$
\frac{\mathrm{v}_{2}^{2}}{2}+\frac{\mathrm{v}_{1}^{2}}{2}=\mathrm{h}_{2}-\mathrm{h}_{1}
$$

According to Bernoulli’s equation,

$$
\frac{\mathrm{P}}{\gamma}+\frac{\mathrm{V}^{2}}{2 \mathrm{~g}}=\text { constant }
$$

As the fluid passes through the diverging portion of the nozzle, according to Bernoulli's equation, pressure drops and velocity increases. Forcing a fluid through the diverging portion doesn't guarantee always that velocity will increase. The state of nozzle is determined by the overall pressure ratio. Back pressure is also responsible for governing the flow in nozzle.

While accelerating through the divergence portion, at a point the acceleration comes to a sudden stop, however, as a normal shock develops at a section between throat and the exit plane which causes a sudden drop in velocity with a sudden rise in pressure. Flow through shock is highly irreversible. As the pressure in the shock region overcomes the back pressure velocity again rises.

Various investigations were done on the flow analysis through both subsonic and supersonic converging diverging nozzles. Khan and Shembharker [1] presented a viscous flow analysis of a convergent divergent nozzle. Du $\mathrm{H}$ et al. [2] conducted a CFD investigation on the nozzle of orifices distributing in different space layers. Besides, Keerthana and 
Rani [3] investigated the flow analysis of annular diffusers. Hussain and Ramjee [4] evaluated the effects of the axisymmetric contraction shape on incompressible turbulent flow. Navier-Stokes Computations of two and three dimensional cascade flow fields were done by Nakahashi [5]. Pandey and Kumar [6] investigated twin jet flow at Mach 1.74 by using CFD analysis. Performance improvement of S shaped diffusers was done by Gopaliya et al. [7] using momentum imparting technique. Kumar G et al. [8] carried out an analysis by using Fluent to design and optimize De Lavel nozzle to prevent shock induced flow separation. Prafulla et al. [9] conducted a CFD analysis on supersonic convergent divergent nozzle. Najar et al. [10] made a comparative study of $\mathrm{K}-\varepsilon$ and Spalart-Allmaras turbulence models for compressible flow through a convergent divergent nozzle.

Finally, sole purpose of this experiment was confined into comparing different properties of these two nozzles with different divergent angle. It was seen that due to variation in divergence angle, properties also varied from each other significantly. Finally the better supersonic nozzle was chosen with high velocity.

\section{MODELLING NOZZLE GEOMETRY}

For this analysis, two simple axisymmetric supersonic nozzles were designed in SolidWorks. Later they had been imported into Fluent for mesh creation as well as flow analysis. The principal difference between these two nozzles is the outlet divergence angle. In Fig. 1 and Fig. 2 outlet divergence angle is $10^{\circ}$ and $20^{\circ}$ respectively. The rest of the dimensions are as following:

- Inlet radius: $30 \mathrm{~mm}$

- Inlet divergence angle: $20^{\circ}$

- Throat radius: $10 \mathrm{~mm}$

- $\quad$ Nozzle length or axis length: $200 \mathrm{~mm}$

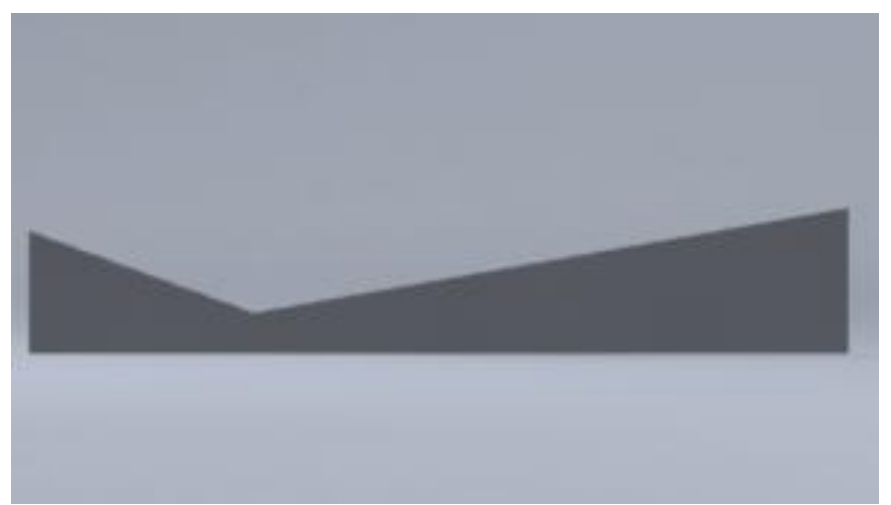

Fig-1: Outlet divergence angle is of $10^{\circ}$

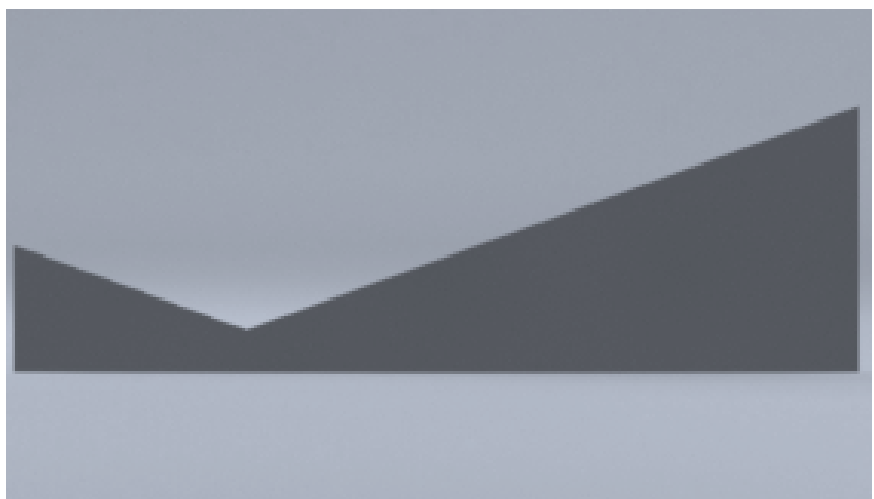

Fig- 2: Outlet divergence angle is of $20^{\circ}$

\section{MESH GENERATION}

A refined mesh for $10^{\circ}$ outlet divergence angle nozzle geometry is represented in Fig 3. Here number of nodes and elements are 1836 and 1717 respectively. Same as, for $20^{\circ}$ outlet divergence angle nozzle geometry, a refined mesh is represented in Fig 4. In this case number of nodes and elements are 2288 and 2163 respectively.

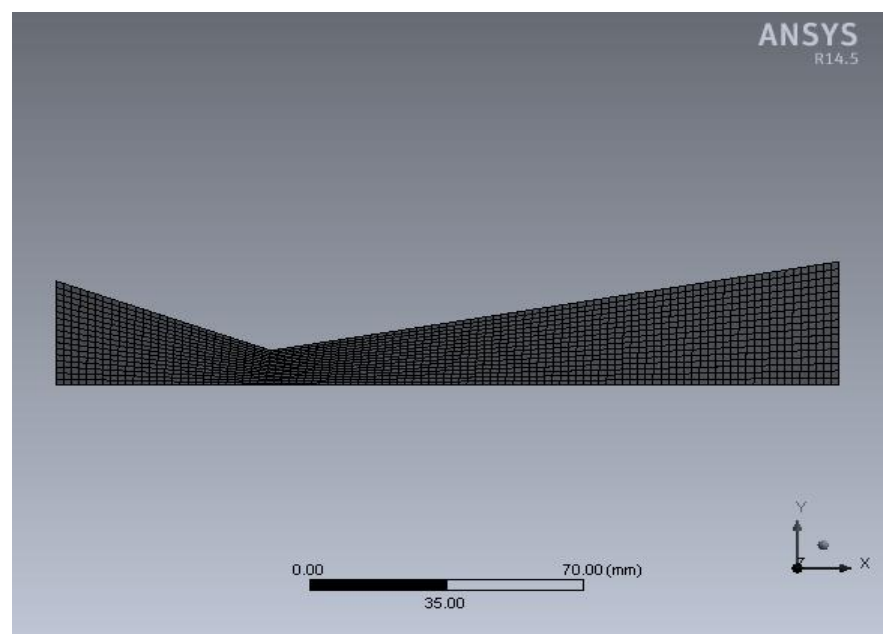

Fig-3: Complete mesh generation of $10^{\circ}$ outlet divergence angle nozzle geometry

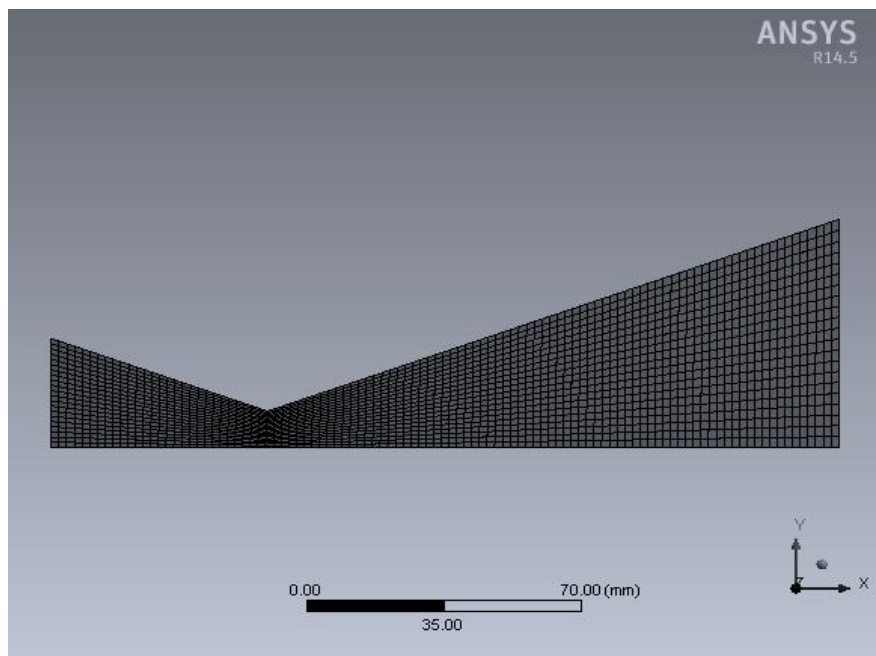

Fig-4: Complete mesh generation of $20^{\circ}$ outlet divergence angle nozzle geometry 


\section{FLOW ANALYSIS}

Before starting the simulation, the boundary conditions were set as following for both nozzles.

Ideal gas was used as containing fluid whereas its specific heat was set as $1006.43 \mathrm{~J} /(\mathrm{Kg} . \mathrm{K})$ and molecular weight was set as $28.966 \mathrm{Kg} /(\mathrm{Kg} . \mathrm{mol})$.

Inviscid laminar flow was considered.

Inlet was set as pressure inlet whose gauge total pressure and supersonic gauge pressure was taken as 18 bar and 8 bar respectively.

- $\quad$ Inlet temperature was set as $300 \mathrm{~K}$.

- $\quad$ Pressure at outlet was set as 2 bars.

- Pressure of operating condition was set as 0 bar.

After initiating the numerical analysis, convergence was obtained after 168th iteration in case of nozzle having $10^{\circ}$ outlet divergence angle and after190th iteration convergence was obtained in case of nozzle having $20^{\circ}$ outlet divergence angle. In Fig 5 and Fig 6 velocity distribution of $10^{\circ}$ outlet divergence angle nozzle geometry and $20^{\circ}$ outlet divergence angle nozzle geometry is shown respectively.
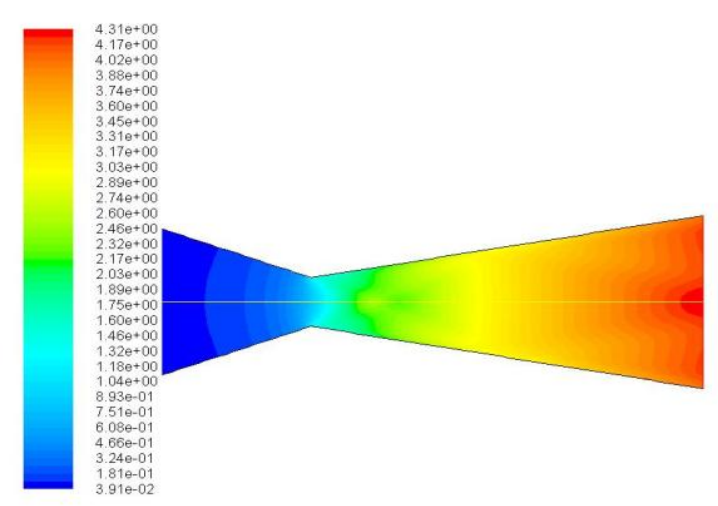

Contours of Mach Number ANSYS Fluent $14.5($ May 30,2014

Fig-5: Velocity distribution of $10^{\circ}$ outlet divergence angle nozzle geometry
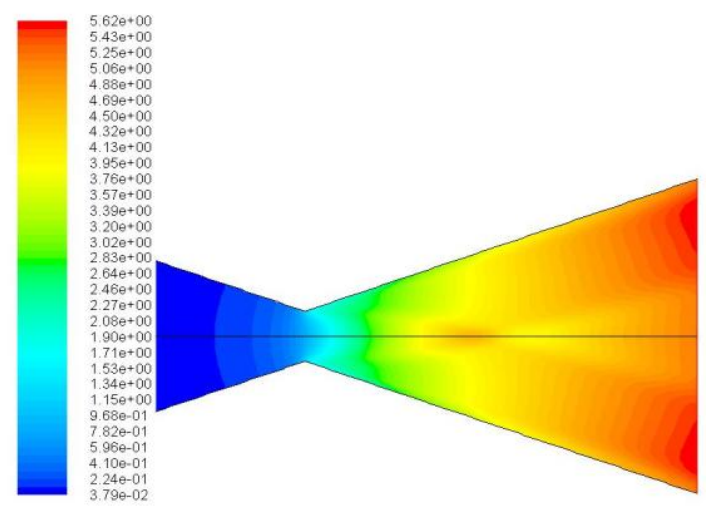

Contours of Mach Number May 31, 2014
ANSYS Fluent 14.5 (axi, dp, dbns imp)

Fig-6: Velocity distribution of $20^{\circ}$ outlet divergence angle nozzle geometry

\section{ANSYS}

In Fig. 7 and Fig. 8 pressure distribution of $10^{\circ}$ outlet divergence angle nozzle geometry and $20^{\circ}$ outlet divergence angle nozzle geometry is shown respectively.

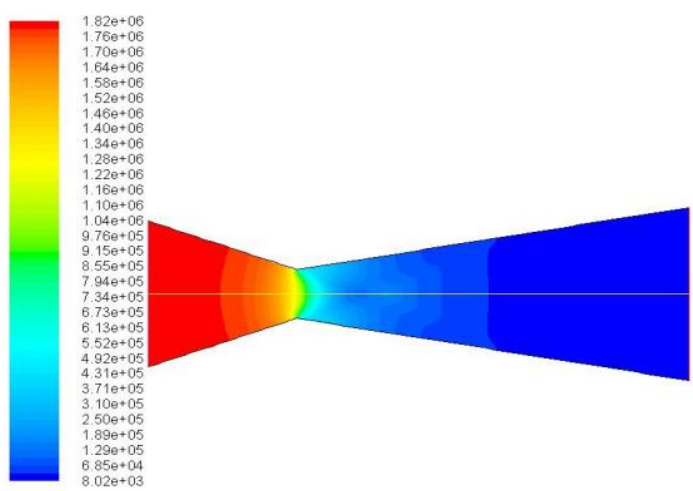

Contours of Static Pressure (pascal) ANSYS Fluent 14.5 May 30, 2014

Fig-7: Pressure distribution of $10^{\circ}$ outlet divergence angle nozzle geometry

ANSYS

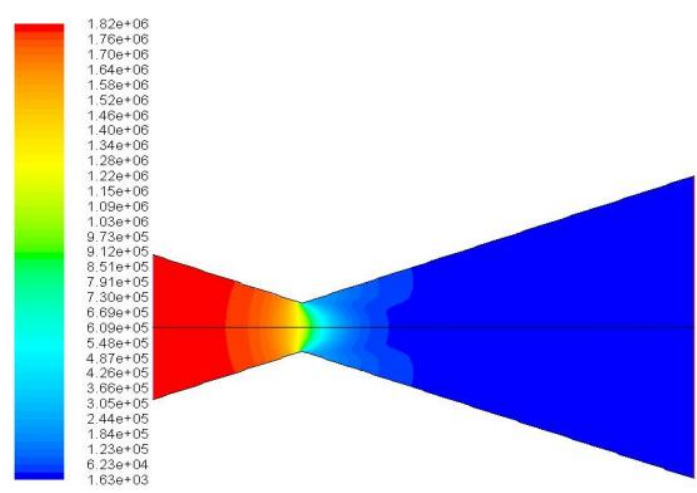

Contours of Static Pressure (pascal)

May 31, 2014
ANSYS Fluent 14.5 (axi, dp, dbns imp)

Fig-8: Pressure distribution of $20^{\circ}$ outlet divergence angle nozzle geometry

In Fig. 9 and 10 temperature distribution of $10^{\circ}$ outlet divergence angle nozzle geometry and $20^{\circ}$ outlet divergence angle nozzle geometry is shown respectively.

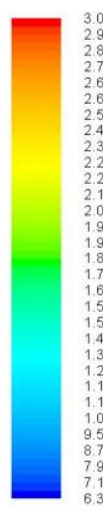

ANSYS

Fig-9: Temperature distribution of $10^{\circ}$ outlet divergence angle nozzle geometry 


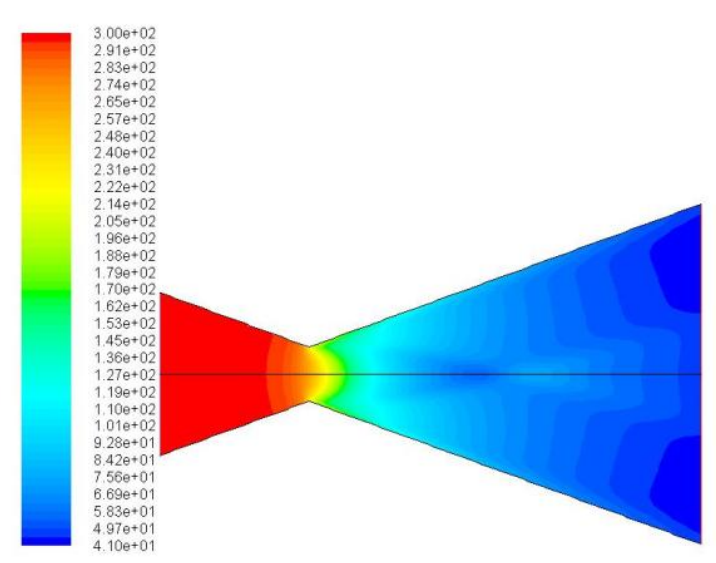

ANSYS

Contours of Static Temperature $(\mathrm{k})$

ANSYS Fluent 14.5

Fig-10: Temperature distribution of $20^{\circ}$ outlet divergence angle nozzle geometry

\section{RESULTS}

In Fig. 11 and 12 graphs were plotted showing variations of Mach number with nozzle length for both nozzles respectively. It is clearly seen the velocity is increasing along with the length of the nozzle for both 10 and 20 degree nozzles. Due to shocking in the nozzle, the velocity decreased for a while but later began to increase as the fluid expanded through the divergent portion.

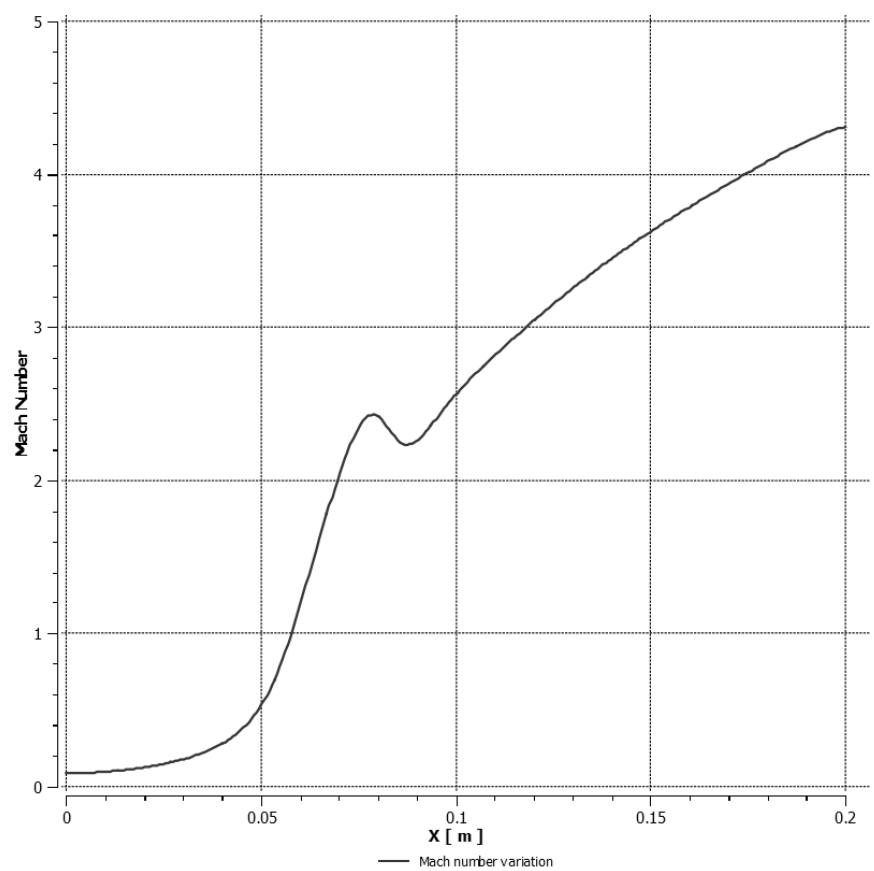

Fig-11: Plot of Mach number versus nozzle length for $10^{\circ}$ outlet divergence angle nozzle geometry

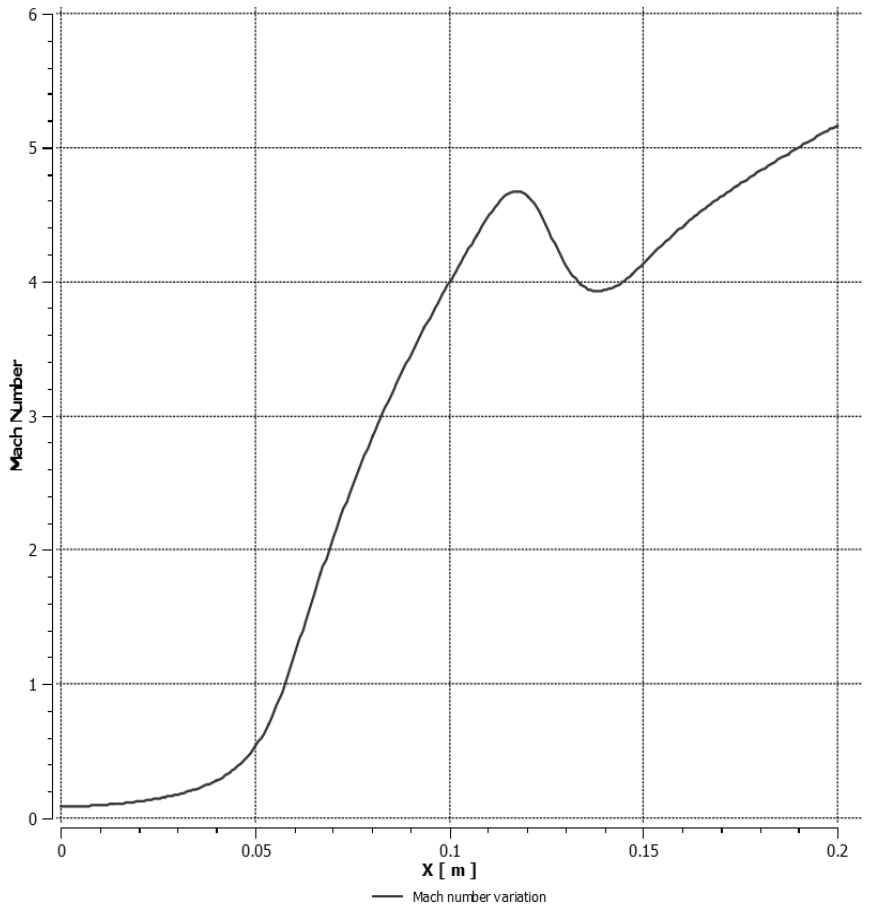

Fig-12: Plot of Mach number versus nozzle length for $20^{\circ}$ outlet divergence angle nozzle geometry

In Fig.13 increase in Mach number with nozzle length for both nozzles was shown simultaneously.

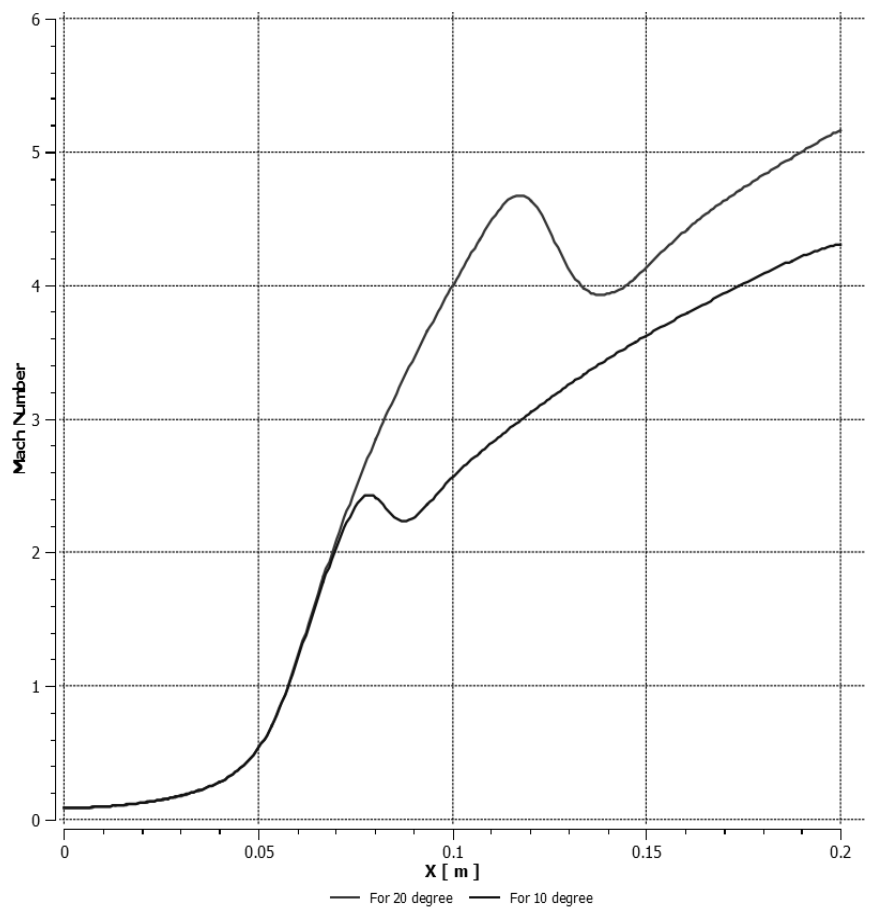

Fig-13: Mach number versus nozzle length for both nozzle geometries.

Fig. 14 and 15 are showing variation of pressure with nozzle length for both nozzles respectively. In both cases, pressure gradually decreased along the length of the nozzle except a slight variation for $10^{\circ}$ divergence angle nozzle. 


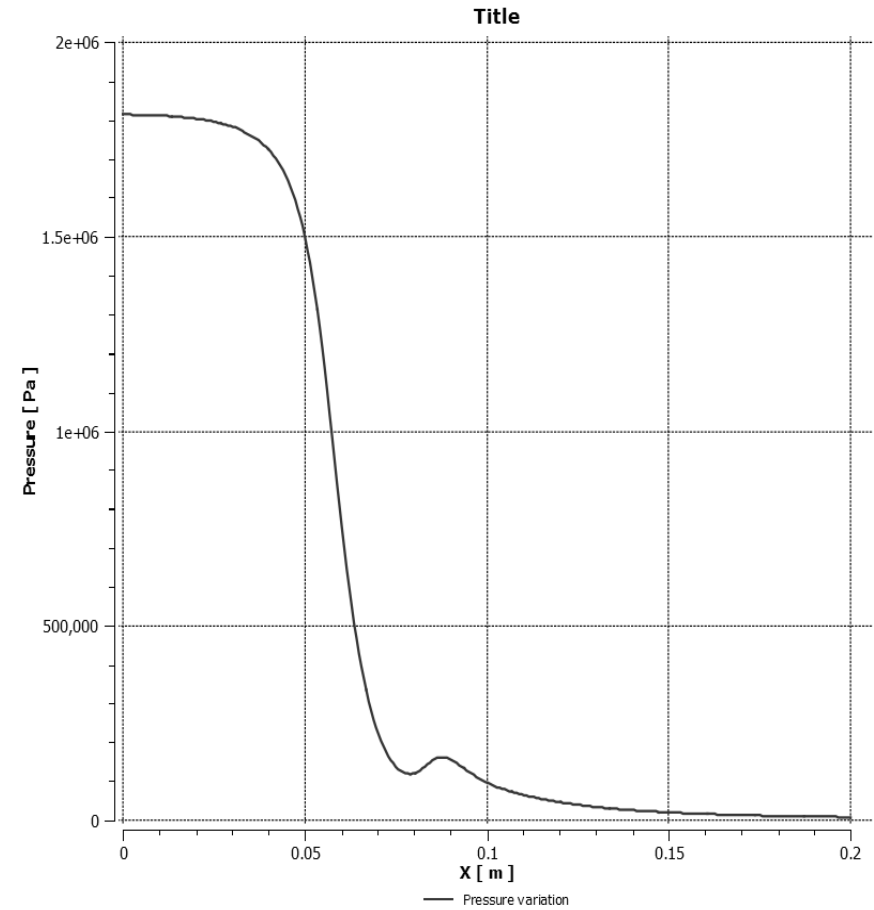

Fig-14: Plot of pressure versus nozzle length for $10^{\circ}$ outlet divergence angle nozzle geometry

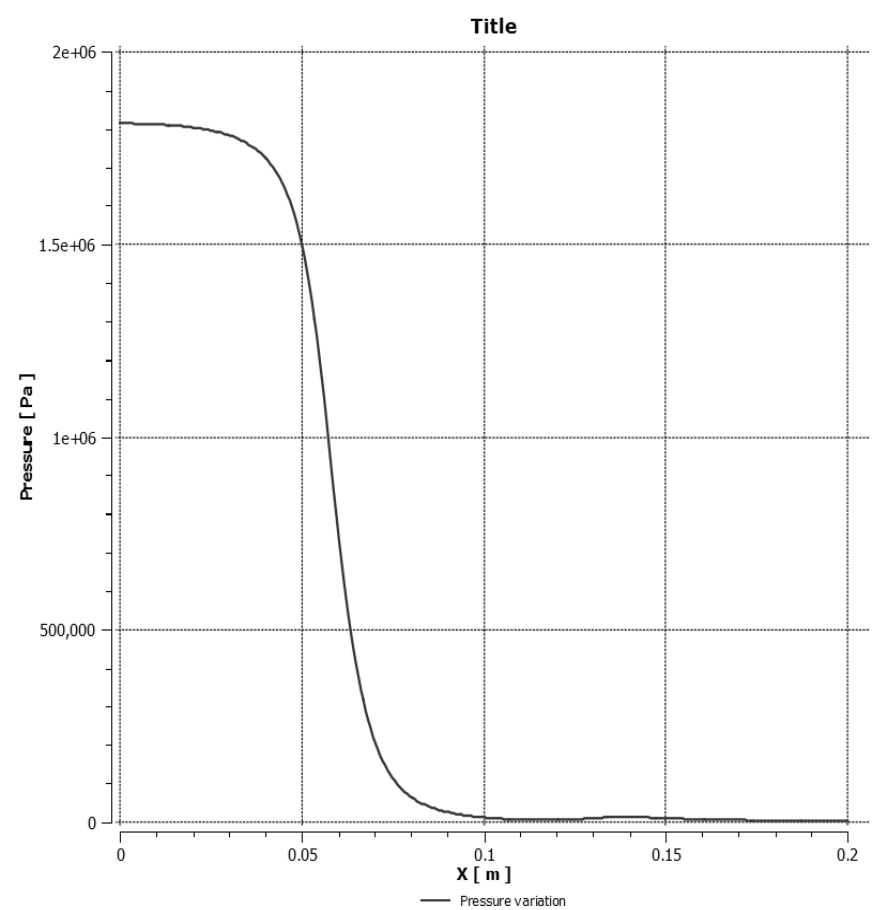

Fig-15: Plot of pressure versus nozzle length for $20^{\circ}$ outlet divergence angle nozzle geometry

In Fig. 16 decrease in pressure with nozzle length for both nozzles was shown simultaneously. As the flow continued along the length of the nozzle, the pressure decreased gradually throughout the nozzle except a slight rise during the shocking. However, the rise was not significant comparing to the total fall in pressure. According to Bernoulli's equation (Eq. 3), pressure decrease as velocity increases along the expansion zone.

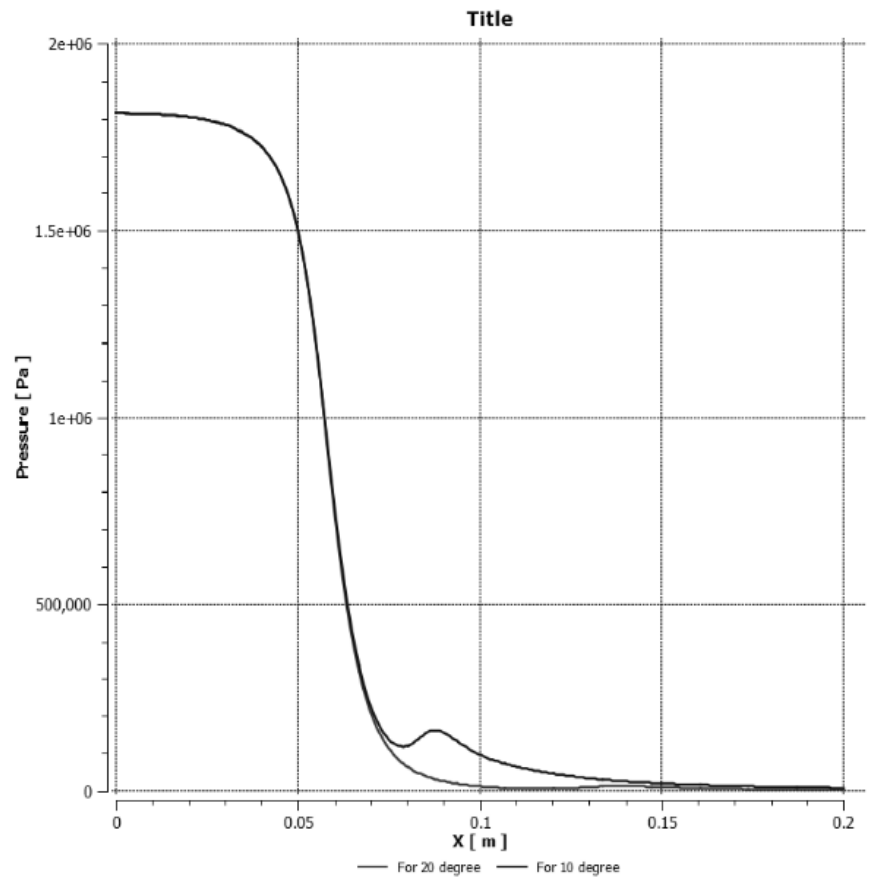

Fig-16: Pressure versus nozzle length for both nozzle geometries.

Variations of temperature with nozzle length for both nozzles are plotted in Fig. 17 and 18 respectively. It is seen that temperature decreased gradually for both 10 and $20^{\circ}$ outlet angle nozzle except a slight increase. The slight increase occurs in the shock zone where rapid change of fluid properties takes place. But the rise in temperature was not significant with respect to the fall in temperature throughout the distance.

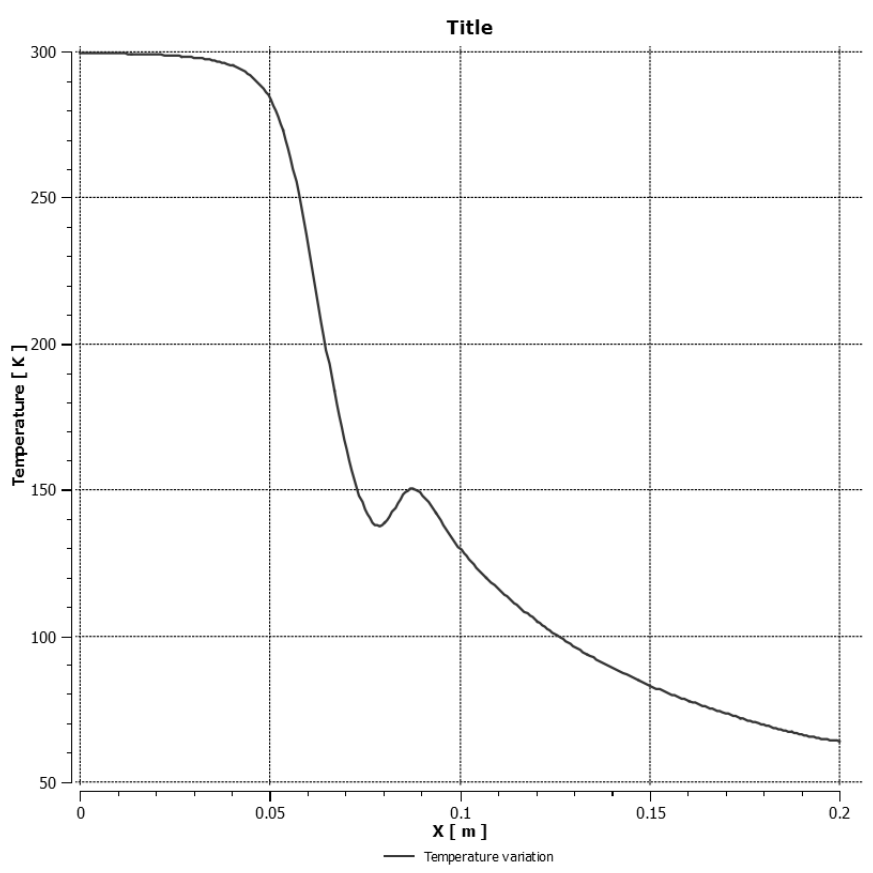

Fig-17: Plot of temperature versus nozzle length for $10^{\circ}$ outlet divergence angle nozzle geometry 


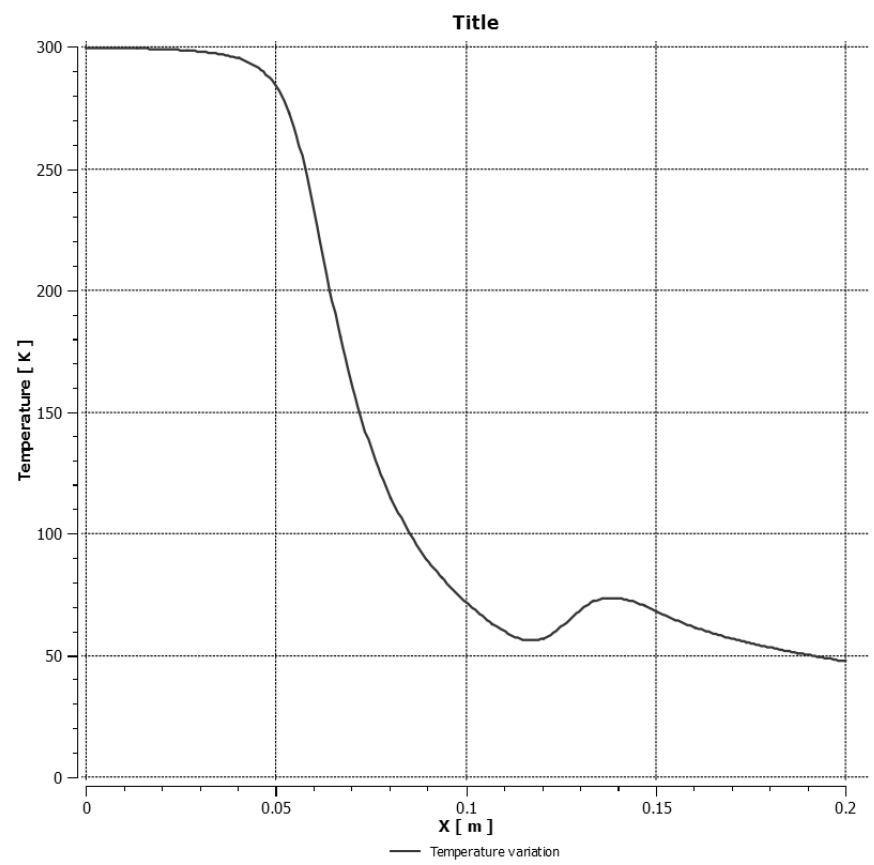

Fig-18: Plot of temperature versus nozzle length for $20^{\circ}$ outlet divergence angle nozzle geometry

In Fig. 19 decrease in temperature with nozzle length for both nozzles was shown simultaneously.

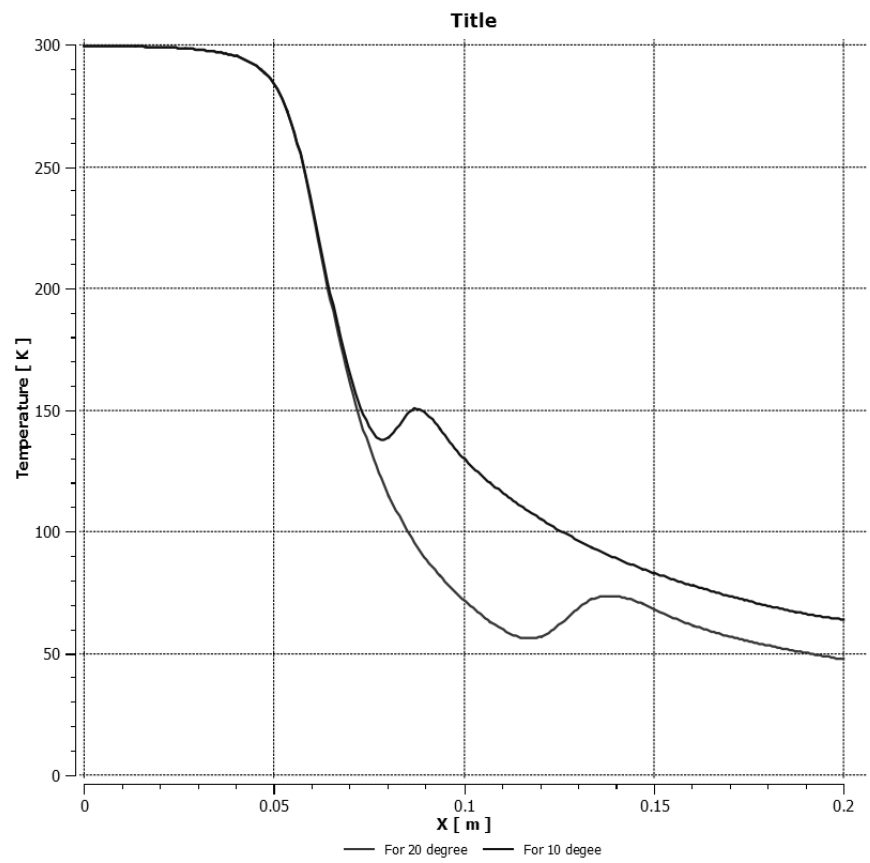

Fig-19: Temperature versus nozzle length for both nozzle geometries.

\section{CONCLUSION}

After successfully completing this simulation based experiment, the decisions were finally confined into the following points.

From the analysis, it is clearly observed that nozzle with outlet divergence angle $20^{\circ}$ gives higher velocity at outlet than the nozzle with divergence angle $10^{\circ}$. The outlet velocities of these nozzles are respectively Mach 5.62 and Mach 4.31. Besides, pressure at outlet for these two nozzles was also found from this analysis. Outlet pressure for $20^{\circ}$ divergence angle nozzle is $1627.53 \mathrm{~Pa}$ and $8022.17 \mathrm{~Pa}$ for nozzle with $10^{\circ}$. Temperature falling rate was larger in nozzle with divergence angle $20^{\circ}$ than the other one. . The values of outlet temperature for $20^{\circ}$ and $10^{\circ}$ nozzles were found as $41.0405 \mathrm{~K}$ and $63.9097 \mathrm{~K}$ respectively. From the analysis it can be concluded that nozzle with $20^{\circ}$ divergence angle gives higher velocity at outlet and lower pressure than the $10^{\circ}$ divergence angle nozzle. So nozzle with $20^{\circ}$ divergence angle is better suited between this two when required for high velocity output. It is also apparent that variations in different properties between these two nozzles occurred due to change in divergence angle from throat towards the expansion zone.

\section{REFERENCES}

[1] A.A.Khan and T.R.Shem bharkar, 2008, Viscous flow analysis in a convergent - Divergent nozzle, International Journal of Computational Engineering Research, IJCERonline, India, Volume 3, No. 5, pp. 5-15.

[2] Du. H, Liu J, Tang J, 2008, A CFD investigation on the nozzle of orifices distributing in different space layers, SAE International, SAE World Congress and Exhibition, USA, 2008-01-0948.

[3] DR. Keerthana and G. Jamuna Rani, 2012, Flow analysis of Annular Diffusers, International Journal of Engineering Research and Application, India, Volume 2, No. 3, pp. 2348-2351.

[4] J Hussain AKMF, Ramjee V, 2010, Effects of the axisymmetric contraction shape on incompressible turbulent flow, Journals of Fluid Engineering, ASME, USA, Volume 98, No. 1, pp. 56-68.

[5] Kazuhiro Nakahashi, 1989, Navier-Stokes Computations of two and three dimensional cascade flow fields, Aerospace Research Central, AIAA, Japan, Volume 5, No. 3, pp. 320-326.

[6] K.M.Pandey, Virendra Kumar, 2010, CFD Analysis of Twin Jet Flow at Mach 1.74 with Fluent Software, International Journal of Environmental Science and Development, India, Volume 1, No. 5, pp. 423-427.

[7] Manoj Kumar Gopaliya, Piyush Jain, Sumit Kumar, Vibha Yadav, Sumit Singh, 2014, Performance Improvement of S-shaped Diffusers Using Momentum Imparting Technique, IOSR Journal of Mechanical and Civil Engineering (IOSR-JMCE), India, Volume 11, No.3, pp. 23-31.

[8] Mohan Kumar G, Dominic Xavier Fernando, R Muthu Kumar, 2013, Design and Optimization of De Lavel Nozzle to Prevent Shock Induced Flow Separation, Advances in Aerospace Science and Applications, India, Volume 3, No.2, pp. 157-165.

[9] Ms. B. Krishna Prafulla, Dr. V. Chitti Babu, Sri P. Govinda Rao, 2013, CFD Analysis of Convergent Divergent Supersonic Nozzle,". International Journal of Computational Engineering and Research, India, Volume 3, No. 5, pp. 5-15. 
[10] Nadeem Akbar Najar, D Dandotiya, Farooq Ahmed Najar, 2013, Comparative Study for $\mathrm{K}-\epsilon$ and Spalart- Allmaras Turbulence Models for Compressible Flow through a Convergent Divergent Nozzle, The International Journal of Engineering and Science, THE IJES, India, Volume 2, No. 8, pp. $8-17$.

\section{BIOGRAPHIES}

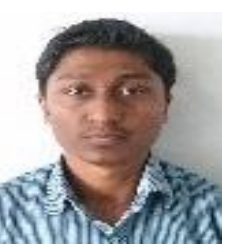

MD. Safayet Hossain was born in 1992 in Chittagong. He is accomplishing BSc in Mechanical Engineering from Chittagong University of Engineering and Technology (CUET) and will complete his course in 2014(expected). His research interests contain Computational Fluid Dynamics, Renewable Energy, Aerodynamics, Heat Transfer and Sustainable Energy.

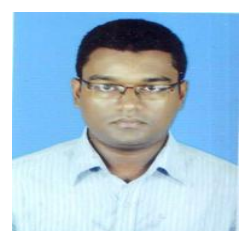

Muhammad Ferdous Raiyan was born in 1990 in Chittagong. He has recently accomplished BSc in Mechanical Engineering from Chittagong University of Engineering and Technology (CUET). His research interests include Computational Fluid Dynamics, Aerodynamics, Heat Transfer, Materials Science, Turbo Machinery and Thermodynamics.

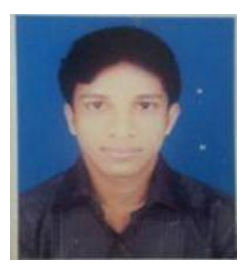

Nahed Hassan Jony was born in 1993 in Chittagong. He is now completing his Bachelor in Mechanical Engineering from Chittagong University of Engineering and Technology (CUET). His research areas are primarily focused on Aeronautical Engineering, Automobile Engineering and Robotics. 